\title{
A Questionnaire System for Institutional Research
}

\author{
Yasuaki Ohira *, Kahori Ogashiwa †, Satoshi Muranaga *, \\ Takahiro Matsumoto *, Hiroo Naitoh*
}

\begin{abstract}
This paper reports a web-based survey system developed to enhance institutional research. The system has basic functions implemented in the open-source web questionnaire system. In addition, this system has functions that enhance institutional research, such as (1) the display of the response rates of each university department, (2) collation of all results obtained (including paper-based surveys), and (3) an analysis of survey results using visual tools. The design considered the importance of a link-up with the authentication server belonging to Yamaguchi University. Thus, graphs may be compiled automatically and in real time to assist a comparison between departments within the university by respective stakeholders. This work provides an overview and a case study of the usage of this system.
\end{abstract}

Keywords: Institutional research, questionnaire system, stakeholders, university evaluation.

\section{Introduction}

Consistent with the University Reform Action Plan, announced in 2013 by the Ministry of Education, Culture, Sports, Science and Technology (MEXT), national universities are currently undergoing a period of reform [1]. Yamaguchi University is also required to set targets for the Third Mid-Term Plan period that started in the fiscal year (FY) 2016, focusing on a regular review of its education and research organization as well as allocation of university resources.

To meet these targets appropriately, fulfilling institutional research is necessary [2]. Institutional research is described in the "Glossary of quality assurance in Japanese higher education" as "a function or department within a higher education institution responsible for the implementation of study and research analysis of information about the institution itself" [3]. One of the functions (methods) of institutional research as decision support is to gather all information on self-assessment and evaluation within the university. Survey methods are particularly important. Perspectives must be collected through surveys, not only from teaching staff and students at the university but also from alumni, students' guardians, employers, and elementary and middle educational institutions. Various surveys have been conducted by departments, with each department attempting its own improvements. Collating and analyzing all these surveys for the university have tended to be difficult. Further, numerous surveys have been conducted through paper-based questionnaires, which are not only expensive but also

\footnotetext{
* Yamaguchi University, Japan

$\dagger$ Utsunomiya University, Japan
} 
generate reduced response rates. In the past, survey results were difficult to accumulate. The current research investigated the functions enhancing institutional research.

The questionnaire system, particularly in relation to university evaluation and institutional research, has received considerable academic attention. Oishi studied the questionnaire survey in university evaluation by hosting a web questionnaire system, developed using an opensource system [4][5][6]. As the response rate of web surveys is generally low, Oishi introduced a reminder function, which proved effective in addressing this specific disadvantage of web surveys. In the case of surveys on stakeholders, Bossé conducted a comparative review of opensource survey tools used in a university setting [7]. The review compared the Moodle module questionnaire [8] and Lime Survey [9], followed by a verification of the effectiveness of the multiple uses of evaluative instruments. Moodle is an open-source learning management system [8], whereas Lime Survey is an open-source survey tool [9]; when used in combination, they enhance data gathering for stakeholders. Thus, various tools may be used for data collection using the survey method; that is, focusing on one tool may be shortsighted. Indeed, tool usability depends on the user (stakeholder). Ease of use for users is greatly improved by using a learning management system that is used on a daily basis. Meanwhile, from an analytical perspective, Lime Survey has a more sophisticated analysis function compared with Moodle. An open-source system is recognized as highly useful for reducing the cost of conducting university questionnaire surveys. However, currently, there are no open-source tools comprising all the functions mentioned above.

Therefore, a system was developed to integrate the required functions. The developed webbased questionnaire system cooperates with the authentication server of Yamaguchi University [10]. This system has basic functions implemented in the open-source web questionnaire system. Moreover, the system enables easy checking of the response rates for each faculty or department in the university. Further, it allows for answers to be limited according to the type of respondent, such as the teaching and administrative staff. This questionnaire system is built into the Yamaguchi University Self-Assessment and Evaluation System [11] [12] and has an important role in the conduct of surveys for university stakeholders. Figure 1 shows the flow of the Yamaguchi University Self-Assessment and Evaluation System. The present study provides an overview and a case study of the use of this system. 


\section{University-wide PDCA system in Yamaguchi University}

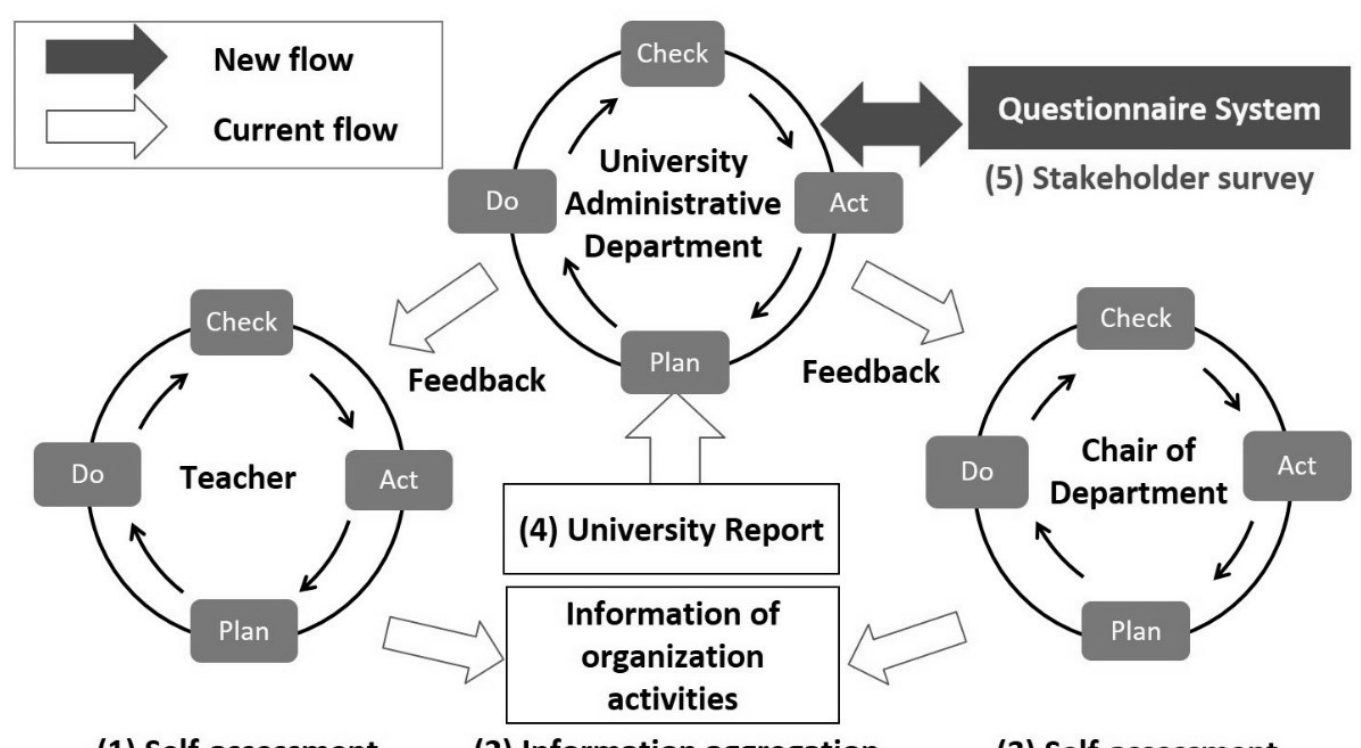

(1) Self-assessment

(2) Information aggregation

(3) Self-assessment

Figure 1: Flow of the Yamaguchi University Self-Assessment and Evaluation System. The university-wide Plan-Do-Check-Act (PDCA) system in Yamaguchi University comprises the following main parts: (1) self-assessment and evaluation (for instructors); (2) information of organizations' activities; (3) self-assessment and evaluation (for department chairs); (4) university report [13]; and (5) questionnaire system (for stakeholders). As shown in this figure, the questionnaire system works as a component of Yamaguchi University's self-assessment and evaluation system. Thus, the original questionnaire system described in this study was developed to meet the specifications of the university.

\section{System Overview}

Figure 2 shows the structure of the questionnaire system. The survey implementer uses a management window to create or delete questionnaires, amend questions, post-disclosure settings, initialize response data, browse response results, and perform other functions. Questionnaires are managed by issuing an account to every implementer. This means browsing or amending questionnaires by other implementers is impossible. 


\section{Structure of questionnaire system}

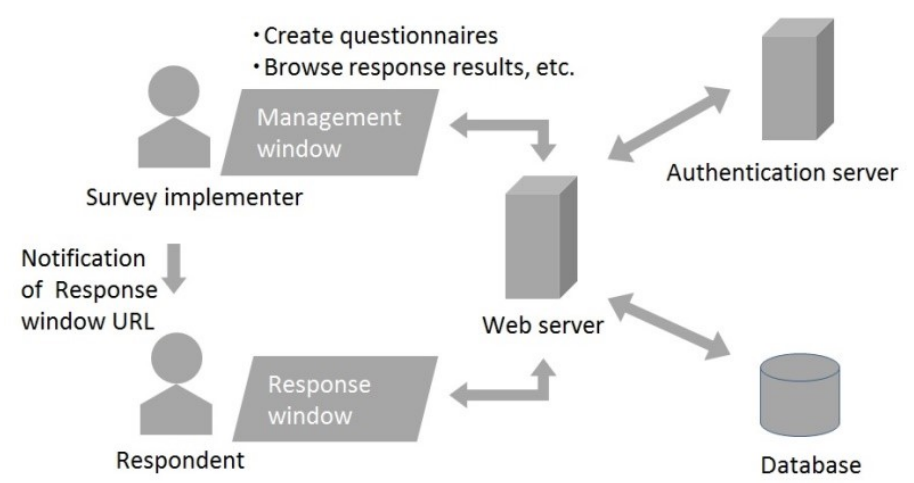

Figure 2: Structure of the questionnaire system. The survey implementer uses a management window to create or delete questionnaires and their content. The respondent uses a response window to respond to the questionnaire.

\section{Questionnaire creation window}

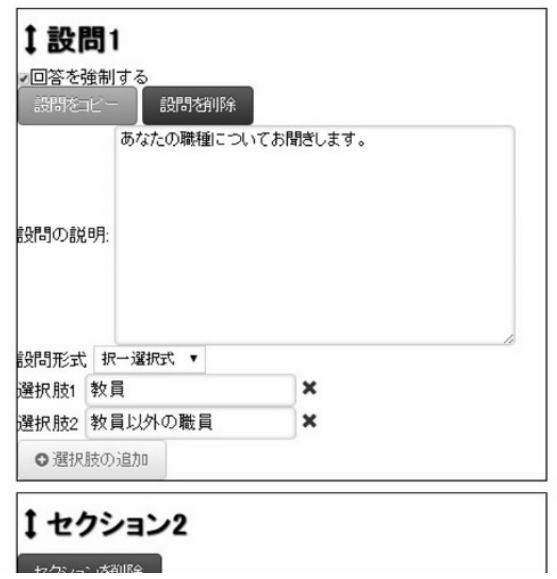

Figure 3: Questionnaire creation window. This tool is used to enter explanatory notes on surveys and to add or delete questions or options.

The questionnaire creation window is depicted in Figure 3. This window is used to enter explanatory notes on surveys and to add or delete questions or options. The use of JavaScript allows for interactive operations, and as long as users have a web browser, HTML or CSS knowledge is not required to be able to create questionnaires intuitively. Available question formats include allowing only one response, multiple responses, and open-ended questions. Questions can be grouped into as many as two levels, thereby facilitating the clustering of questions that share the same topic. Moreover, the creation window allows the user to create questions that require responses. 


\section{Response token management window}

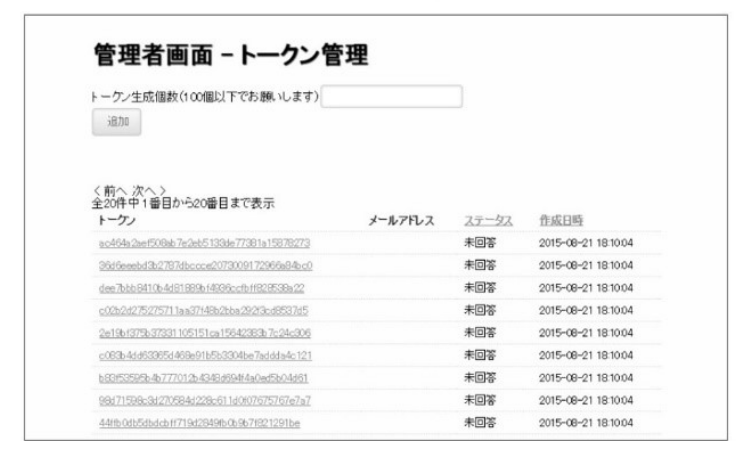

Figure 4: Response token management window. This setting is available for issuing any number of tokens and limiting respondents to only those who have been given a token. This is effective to prevent a single respondent replying to a survey multiple times.

\section{Response rates window}

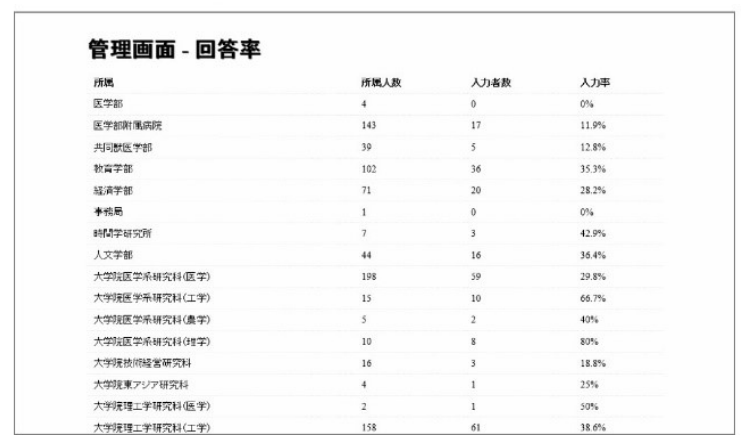

Figure 5: Response rates window. Response rates are displayed for each faculty or department in Yamaguchi University.

Once a questionnaire has been created, respondents are sent an email containing the URL of the response window. Respondents open the response window in their browser and complete the questionnaire. As the URL contains a randomly generated hash value, gaining access to a response window for a survey of which a respondent has not received notification is virtually impossible.

The disclosure settings allow for a password to be set for a questionnaire. This function prevents prank responses even when the URL has been sent to an unspecified but large number of users. Further, a setting is available for issuing any number of tokens and limiting respondents to only those who have been given a token. These tokens (hereafter referred to as response tokens) are random hash values and used by a user authorized to use computer services to aid authentication. Response tokens are generated on the response token management window. The response token management window confirms which tokens have been used in responses, thereby allowing its use in studying response rates. The response token management window is depicted in Figure 4. 
Further, a link-up with the authentication server provided by the Media and Information Technology Center at Yamaguchi University enables settings that require a user to enter a Yamaguchi University email account and password when opening a response window, or enables the user to limit respondents to teaching or administrative staff [14]. If this authentication function is enabled, and if survey respondents are limited to teaching or administrative staff, the response rates can be determined for each department on the basis of data obtained from the authentication server. Figure 5 shows the response rates window.

Paper-based surveys are required for respondents who are not set up in a network environment or have difficulty responding through a PC. Results obtained through paperbased surveys can be entered into an Excel file; an interface window is provided to upload the file. This method allows for the collation of results obtained online and in person. An Excel file for this purpose can be generated and downloaded from the management window.

Response results can be confirmed in pie charts in the response results browsing window. Figure 6 shows an example of the response results window for a question having two options, only one of which must be selected. On the assumption that report writers prefer to create their own graphs while reporting response results, the system also provides an option to download results for each question in a comma-separated values format. Further, to guarantee respondent anonymity, the responses and personal information of respondents cannot be viewed.

\section{Response results window}

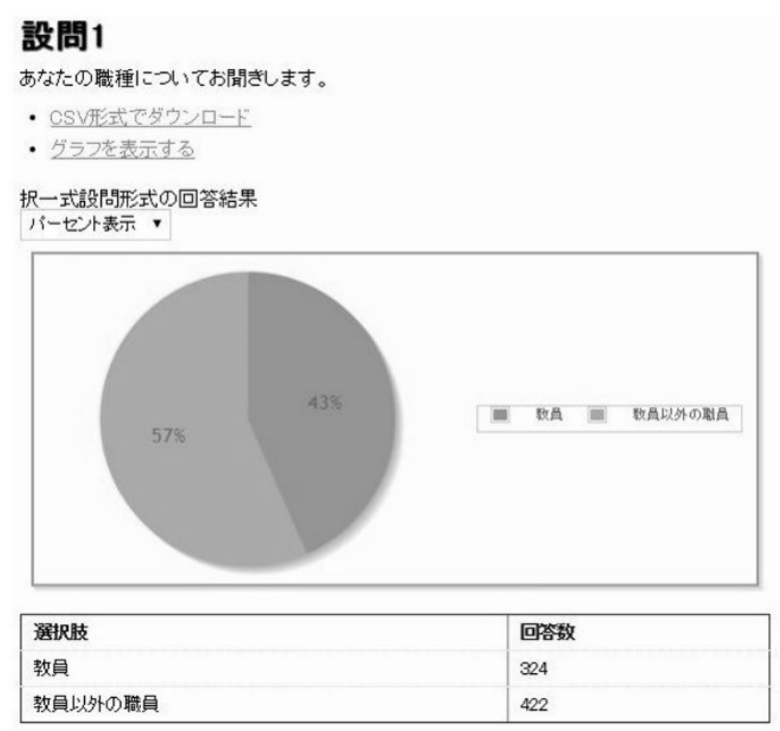

Figure 6: Response results window. Response results can be confirmed in pie charts. The figure shows an example of the response results window for a question with two options, only one of which must be selected. The system also provides an option to download results for each question in a comma-separated values format. 


\section{Case Study}

The present study outlined the development of a system for easy web-based survey implementation. The system enables people without HTML or CSS knowledge to create surveys easily, analyze survey results using graphs, and confirm the response rates. Further, the system has functions for limiting answers to those from teaching or administrative staff, as well as for checking the response rates for each faculty or department. The case study involved using the system for a survey among university personnel and teaching staff to determine their awareness of university objectives and policies. We used this system and conducted the following three questionnaire surveys: (1) awareness survey of university objectives and policies, (2) survey to graduates, and (3) homecoming survey. The respondents of survey 1 were teaching or administrative staff and those of survey 2 and 3 were graduates. According to the results for survey 1 (awareness of the basic principles of the university), approximately $90 \%$ people "know" [15]. These survey results were used for self-assessment reports and the evidence data on which they are based, such as improvement of university activities. Topics for a future study include incorporating a function for visualizing changes over time on the basis of surveys conducted regularly over long time periods.

\section{Comparison with Similar Systems and Discussion}

Web questionnaire systems play a significant role to improve institutional research in universities, where both paper-based questionnaires and web systems are employed. As is well known, a web questionnaire system has more advantages than a paper-based. For example, the web system is able to provide faster response rates, decrease of sending a reminder to participants, and decrease of disturbing analysis of participants' data. According to the previous study [12], it is important to compare departments in the university to enhance institutional research. Therefore, we designed and developed some functions, such as "user authentication," "link-up with the authentication server," and "visualization of comparison results of each department," capable of an automatic performance of a series of operations of a questionnaire survey. As shown in Table 1, it is possible to develop some of the functions for institutional research by combining (integrating) and customizing the functions of a general web questionnaire. However, it is difficult to meet all specifications for institutional research. Therefore, we carefully selected only the functions that are important (necessary) for institutional research and developed them uniquely. As a result, the proposed system realizes simple operability and high functionality. Data analysis and visualization are required not only for data analysis for periodic survey reports but also for decision-making in universities. To predict the future on the basis of historical data plays a significant role in making decisions. This system also has a function to accumulate past data. 
Table 1: General functions of a web questionnaire system and important functions for institutional research. Bold letters show important functions for institutional research.

\begin{tabular}{|c|c|c|c|c|c|}
\hline No. & Function & Contents & Lime Survey & $\begin{array}{c}\text { Moodle } \\
\text { (questionnaire } \\
\text { module) } \\
\end{array}$ & $\begin{array}{c}\text { The proposed } \\
\text { system }\end{array}$ \\
\hline \multirow[t]{2}{*}{1} & \multirow{2}{*}{$\begin{array}{l}\text { System } \\
\text { administration }\end{array}$} & User authentication & ○ & $\circ$ & $\circ$ \\
\hline & & $\begin{array}{l}\text { Link up with } \\
\text { authentication } \\
\text { server }\end{array}$ & O & ○ & O \\
\hline \multirow[t]{3}{*}{2} & \multirow[t]{3}{*}{$\begin{array}{l}\text { Questionnaire } \\
\text { administration }\end{array}$} & $\begin{array}{l}\text { Create a } \\
\text { questionnaire page }\end{array}$ & O & ○ & o \\
\hline & & Create a question & 0 & ○ & ○ \\
\hline & & $\begin{array}{l}\text { Setting of input } \\
\text { subject faculty } \\
\text { (teacher, student) }\end{array}$ & --- & ० & ○ \\
\hline \multirow[t]{2}{*}{3} & \multirow[t]{2}{*}{ Mail } & Information mail & ○ & ○ & ○ \\
\hline & & Remind mail & oAuto & --- & oManual \\
\hline \multirow[t]{4}{*}{4} & \multirow[t]{4}{*}{$\begin{array}{l}\text { Aggregate, } \\
\text { analysis }\end{array}$} & $\begin{array}{l}\text { Real time } \\
\text { aggregation }\end{array}$ & O & o & O \\
\hline & & Graph display & 0 & 0 & 0 \\
\hline & & $\begin{array}{l}\text { Output of analysis } \\
\text { data }\end{array}$ & ○ & ○ & ○ \\
\hline & & $\begin{array}{l}\text { Visualization of } \\
\text { the result of } \\
\text { comparing } \\
\text { departments }\end{array}$ & --- & --- & o \\
\hline 5 & $\begin{array}{l}\text { Data collection } \\
\text { (accumulation) }\end{array}$ & $\begin{array}{l}\text { Store paper-based } \\
\text { questionnaire } \\
\text { results in a } \\
\text { database (such as } \\
\text { accumulation of } \\
\text { past data). }\end{array}$ & --- & --- & o \\
\hline
\end{tabular}

\section{Conclusion}

The present study outlined the development of a system for easy web-based survey implementation. The system enables people without HTML or CSS knowledge to create surveys easily, analyze survey results using graphs, and confirm response rates. Further, the system has functions for limiting answers to those from teaching or administrative staff, as well as for checking the response rates for each faculty or department. We conducted a case study on using the system for a survey among university personnel and teaching staff to determine their awareness of university objectives and policies.

Topics for further study include incorporating a function for visualizing changes over time on the basis of surveys conducted continually over long time periods. 


\section{Acknowledgement}

This work is supported by the Ministry of Education, Culture, Sports, Science and Technology, Grant-in-Aid for Scientific Research (C) (JSPS KAKENHI Grant Number JP15K01071).

\section{References}

[1] Ministry of Education, Culture, Sports, Science and Technology Japan, The University Refo rm Action Plan 2013 (in Japanese), 8 November 2016; http://www.mext.go.jp/a_menu/kout ou/houjin/1341970.htm.

[2] J.L. Saupe, Functions of Institutional Research, 2nd Edition, Association for Institutional Research, 1990.

[3] Glossary of quality assurance in Japanese higher education, National Institution for Academic Degrees and Quality Enhancement of Higher Education, April 2016.

[4] T. Oishi, "Effects of reminders in questionnaire survey," Proc. 3rd International Conference of Institutional Research and Institutional Management, 2014, pp. 391-394.

[5] T. Oishi, M. Mori, and E. Takata, "Reminder effect and data usability on web questionnaire survey for university students," Proc. 2015 International Conference New Perspectives in Science Education, Edition 4, 2015, pp. 441-444.

[6] T. Oishi, N. Kuwano, E. Takata, "Comparison between two web questionnaire surveys," 5th IIAI International Congress on Advanced Applied Informatics (IIAI-AAI), Kumamoto, 2016, pp. 497-500.

[7] C. Bossé, C. Ives, and D. Briton, "Using open source survey tools for qualitative inquiries on educational development at a distance online university," 10th European Evaluation Society Conference, O-297, 2012.

[8] Moodle, 10 November, 2016; https://moodle.org/,.

[9] Lime Survey, 10 November, 2016; https://www.limesurvey.org/.

[10] Y. Ohira, K. Ogashiwa, S. Muranaga, T. Matsumoto, and H. Naitoh, "A development of a questionnaire system for institutional research," 5th IIAI International Congress on Advanced Applied Informatics (IIAI-AAI), Kumamoto, 2016, pp. 505-508.

[11] University Evaluation Department, Yamaguchi University, "Self-assessment and evaluatio n" (in Japanese), 8 November, 2016; http://committee.ue.yamaguchi-u.ac.jp/New_HomePag e/jikotennkennhyoka-system.html.

[12] Y. Ichikawa, K. Ogashiwa, M. Suzuki, T. Matsumoto, and H. Naitoh, “An overview of YUSE, Yamaguchi University Self-Evaluation System, in relation to university selfevaluation activities in the first mid-term goals period and the first half of the second period" (in Japanese), Journal for Academic Computing and Networking, no. 16, 2012, pp. 3-14. 
[13] FY 2015 Yamaguchi University Report (in Japanese), 16 November, 2016; http://committe e.ue.yamaguchi-u.ac.jp/New_HomePage/27nenndo-hakusyo.pdf.

[14] Y. Hisanaga, J. Kariya, and H. Miike, "The introduction of unified authentication in Yamaguchi University" (in Japanese), Journal for Academic Computing and Networking, no. 10,2006 , pp. $55-62$.

[15] Yamaguchi University Self-Assessment Report (in Japanese), 16 November, 2016; http://c ommittee.ue.yamaguchi-u.ac.jp/New_HomePage/ninnsyohyoka-jikotennkennhyokasyo-h2 7.pdf. 